\title{
MAPPING THE USER EXPERIENCE: DEVELOPMENT OF A VALIDATED INSTRUMENT FROM THE PLANS AND SCRIPTS OF THE COMPUTER COMMUNITY OF PRACTICE
}

\author{
Pat Lehane \\ Defence and Industrial Systems \\ University of South Australia \\ Australia
}

\begin{abstract}
A plethora of surveys for assessing computer use for usability or technology acceptance exist today. This article discusses the Systems Acceptance Indicator, a validated survey instrument for assessing the user experience from a cognitiveergonomic perspective. The action research discussed in this paper utilized grounded theory analysis to establish the data-driven emergent theoretical constructs that provided the system acceptance categories (criteria) for the survey. These data-driven emergent theoretical constructs were the basis for the proposed theoretical abstraction hierarchy of the survey criteria. Principle component analysis of the survey data produced an abstraction hierarchy identical to the theoretical model. This result confirmed the alignment of the human-computer interaction theoretical constructs with the data-driven emergent theory. The intent behind the human-computer interaction theoretical rationale for the emergent abstraction hierarchy was to provide a consistent and repeatable interpretation of the user response to the survey.
\end{abstract}

Keywords: validated survey, interaction design, community of practice, intuitive, $\mathrm{HCI}$, user experience, cognitive ergonomics.

\section{INTRODUCTION}

This article is focused on the emergent theoretical constructs that shaped a survey for system acceptance. The survey, the System Acceptance Indicator (SAI; Lehane, 2012a), was developed for the specific purpose of assessing system acceptance and the user experience (UX) from the pragmatic perspective of a human-computer interaction (HCI) practitioner. The typical numerical index or summarized qualitative description of the analysis of the participants' responses to a survey can be less than useful in regard to how the HCI practitioner should proceed in regard to the users' perceptions of the system - an uncovering of any useful design and development concepts - or action based on the solicited user feedback. Where this survey differs is that the analysis practice associated with the SAI (Lehane, 2012b) is intimately linked to the proposed theoretical rationale derived from the underlying HCI precepts.

(C) 2012 Pat Lehane, and the Agora Center, University of Jyväskylä

URN:NBN:fi:jyu-201211203033 
The theoretical constructs discussed here are 12 criteria that emerged from a grounded theory analysis (Dick, 2005; Glaser, 1994, 1998) of notes collected from focus group interviews with users as part of the survey. The purpose of the survey was to provide a general indication of the users' system familiarity based on their then-present usage and their leverage of prior knowledge and experience. The concept of intuitive computer use by a community of practice (Vygotsky, 1978) provides the foundation for interpreting the responses to the survey (Lehane, 2010). In particular, the concept of leverage of prior knowledge, expressed as previously learned or scripted behavior patterns, was an important concept in this study. Scripted behavior (Bødker, 1991; Suchman, 1987) is a key concept used for considering a design as intuitive and for the subsequent resolution of arising use issues.

When the survey responses are viewed as a time series comprising a benchmark, transition, and familiar use, the UX is presented in terms of HCI design concepts. The strength of the survey is that the time series of responses can show changes in high-level user perceptions, and an associated remedial program can be implemented if a shortfall is identified. In such situations, usability engineering concepts (Nielsen, 1993) are not applied generally at the process level as a learned behavior; rather, they are applied at the keystroke level as the conscious operationalization of the scripts, which establish the users' expectations for interacting with the system. That subtle variation was observed during the investigation into users' interaction with large enterprise systems with limited or no customizations (Lehane, 2012a).

In this article on intuitive design, I discuss the underlying theoretical rationale developed for understanding and implementing concepts associated with plans and scripted behavior in system design. The HCI theoretical rationale addresses familiar concepts, such as usability (Nielsen, 1993), distributed cognition (Hutchins, 1995, 2000), and activity theory (Bødker, 1991; Nardi, 1996a; Vygotsky, 1978), before dealing with extensions made to these existing models. I then introduce the innovative interpretation that the use of these extensions allows.

\section{THEORETICAL RATIONALE FOR EMERGENT CONSTRUCTS}

At the start of the research project, it became very clear to me, as the systems analyst, that the way the users described their experiences mirrored issues typical of UX, but differed significantly in regard to the application of specific terms and concepts as compared to those used in the HCI literature. Thus, if an appropriate direction toward understanding the UX was to be found, I realized I needed to reconcile these discrepancies.

Systematic note-taking was the initial response, followed by a process of coding the concepts and comparing them to HCI literature. (A fuller description of the process can be found in Lehane, 2012b.) Resulting from this iterative process, concepts from a number of paradigms were brought together to form the contextual interpretation presented here in explaining the emergent theoretical construct, which is the classification criteria of the survey. The concepts behind these criteria characterize the users' descriptions of their computer-use experiences and included, but was not limited to, activity theory (Bødker, 1991; Nardi, 1996a; Vygotsky, 1978), situated action (Suchman, 1987), distributed cognition (Hutchins, 1995, 2000), usability engineering (Nielsen, 1993), soft systems modeling (Checkland, 1999; Checkland \& Holwell, 1998), cognitive systems engineering/cognitive work analysis (Rasmussen, 1994; Vicente, 
1999), scientific management (Taylor, 1911), and the unified theory of acceptance and use of technology (Venkatesh, Morris, Davis, \& Davis, 2003).

One premise for grounded theory is that the theory is concealed within the data. To reveal this theory implicit in the data, the data collection, analysis, and theory formulation are approached in an iterative process. Thus, the research process is an inductive investigation in which relevant data is systematically gathered and analyzed. The objective is not to test a theory but to observe and seek further emergence that will build onto the existing theory (Dick, 2005). The use of grounded theory as the vehicle to expose emergent theory from the research data ensures that the data are not forced unquestioningly to conform to espoused theory (Carroll, 1991; Glaser, 1998; Kuutti, 1996).

The final phase of the implementation of grounded theory requires a propositional theoretical base, developed from the data collection and analyses, which is used to develop criteria for the theoretical sampling and associated classification method (Dick, 2005; Glaser, 1994, 1998). The SAI survey (see Lehane, 2010, 2012b) is that final stage of my grounded theory analysis. The analysis of this final-stage data was to confirm or refute the relationships between categories and, where applicable, to limit the relevance of the categories in the proposed theoretical constructs. The discussion about the influence these paradigms have on the UX and system acceptance presented here accounts for the HCI theoretical rationale used to interpret the users' responses to the survey.

\section{Extensions to Existing Models}

While developing the theoretical rationale to explain the emergent constructs and to question categories as presented in this article, it was considered opportune to include in the original models the theoretical tenets not represented but which were important to the interpretation of the survey results. Extensions were made to the original models of activity theory (Engeström, cited in Kuutti, 1996; Hasan, Gould, Larkin, \& Vrazalic, 2001; Vygotsky, 1978) and usability engineering (Nielsen, 1993). In addition, soft systems modeling (Checkland, 1999) was used to develop a theoretical rationale for the abstraction hierarchy of the usability engineering criteria; concepts from cognitive HCI were used to operationalize activity theory. This restructuring helped establish the theoretical rationale for the survey criteria, which are the grounded theory sampling classes.

Two concepts that describe activity theory principles are context and consciousness (Nardi, 1996b). I interpret the Vygotsky/Engeström model as being focused on the context. Both context and consciousness are important to activity theory as it is applied in this article and to practice in general. Consequently for the development of the theoretical rationale, consciousness as orientation was mapped into the model.

The pictorial representation of Nielsen's model of the attributes of system acceptance in usability engineering did not include functionality (Nielsen, 1993, p. 25), yet the criteria usability and utility qualify functionality. Functionality is a key concept in a system's analysis, design, and practice. Therefore, the human factors definitions of tasks and functions were used to establish functionality in Nielsen's model (Lehane, 2012a).

Soft systems modeling has two important pairs of concepts: emergence and hierarchy, and communication and control (Checkland, 1999, p. 75). The application of these principles 
to usability engineering, in conjunction with the incorporation of functionality, allowed the system acceptance criteria to be structured theoretically into an abstraction hierarchy.

The tenets of activity theory are broad-scope concepts with high-level abstract explanations. Consequently, minimal detail on their implementation is available. Thus, HCI models and theories were used, where applicable, as methodologies to provide the low-level implementation detail missing from activity theory. The incorporation of the HCI concepts into the structure of the activity theory model provided pragmatic methodologies to articulate activity theory within the HCI domain. By incorporating these methodologies in this study, activity theory subsumed those cognitive HCI models and theories.

Finally, as a user requirement, system designers are frequently asked to "make it intuitive." This article surfaces one way in which this requirement can be considered in design. That solution, scripted behavior based on the practice of a use community, emerged from the theoretical backdrop developed to understand the role and influence of the community of practice on the users' day-to-day computer-use experiences. The following discussion outlines the implications and outcomes of applying the communities of practice paradigm to the analysis and interpretation of the user experience, as exposed by responses to the SAI.

\section{The User Experience}

The integration of information and communications technologies (ICTs) into everyday activities at work, at home, and at play has made computer use a holistic experience. People now talk of their UX as they engage in the multiple activities that compose their days. Each use instance of engaging with technology contributes to the users' UX, building up a mosaic of experiences across a spectrum of occurrences. Each experience results in a script of contextualized actions appropriate to the environment and the task. Later these scripts can be recalled as planned activities to resolve user interaction issues.

Concept-wise, the challenge today is not only for design to build on and improve the UX, but also to monitor and evaluate the UX across myriad contextual situations made possible by the enabling technologies. To do this, both design and evaluation need to consider aspects of the situated activity and associated scripted behavior in an integrated manner, so as to present familiar artifacts and use experiences to the user. Users who recognize familiar artifacts and their use possibilities are led to a UX in which they perceive the system to be easy to learn and use. This result is the intuitive design presented in this article.

\section{Cognition in Interaction Design}

In 1987, Suchman wrote about purposeful action and the context that defined the meaning of such actions. Suchman stated that artifacts built on a planning model confuse the concepts of plans and situated action. Plans are based on previously experienced conditions, as well as the consequences of actions that accounted for the actions in a plausible way. Plans and goals do not provide a solution to the problem; they simply restate it. The term situated action underscored Suchman's view that every course of action depends upon its material and social circumstances. These scripts are extremely numerous, one for almost every occasion (e.g., a restaurant script, a birthday script, etc.). Each situation has its "plan" comprising ordered action sequences, with each action producing the conditions that enable the next action to occur. The integration of 
situated action into an activity is an emergent property of moment-by-moment interactions, based on previous experiences, between the individual and the environment of his/her actions.

In the same way that buying a cup of coffee at Starbucks is not the same as buying a cup of coffee at McDonalds, the environment and context are distinct constructs. People behave differently in response to the conditions because of various learned responses, that is, scripts. Plans associate intentions with action sequences, and scripts associate action sequences with typical situations, thus forming the precursors to an intuitive response.

Similarly, Bødker (1991) used activity theory concepts in discussing the context of human activity that defined the meaning of such activities. The individual activity is conducted through actions that take place at a nexus of time, space, and specific intentions. An action is conducted through one or more operations. Operations are the sensorimotor deeds that a human performs, without consciousness, in a specific situation to fulfill the conscious actions. The appropriate action is triggered by the material conditions, that is, the script. If these triggers do not exist, then the action is not operationalized and the action has to be performed with conscious attention to detail.

In the workplace, which is a controlled community of practice, individuals share the practice at the same time as they are participating in the activities constituting the practice. Through learning (i.e., special learning activities or in daily work activities), individuals come to possess a context-sensitive repertoire of operations to be used in a specific activity. In the workplace, practice is passed from one person to another. The novice becomes familiar with the artifacts, interactions, and business processes necessary to produce the required work outcomes. Issues arise when the existing system is replaced by another that has the same business capabilities but a different look and feel. In such cases, the interaction design is completely changed by the unfamiliar screen layout and artifacts. Significant changes to the look and feel of a system or a process within a system can produce a replacement that is counter to the design goal of producing an intuitive system. Consequently, the material conditions, learned from past experiences and executed as scripts, are the elements that must be transported to the screen and presented on the new interface for intuitive interaction design.

\section{Emotional User Response in a Cognitive World}

Norman (2004) assigned three levels of processing to the human brain: visceral, behavioral, and reflective. Visceral is the instinctive processing that instantaneously makes a value judgment on what is good or bad (physically, mentally, and emotionally) and sends appropriate signals to the muscles and the brain. Affective processing, which includes emotional response, starts with the visceral. Everyday behavior, best described as skilled or operationalized activity without conscious consideration of the activity, is the behavioral level. Finally, the reflective level involves cognition, the contemplative processing of past, present, and proposed experiences.

The lowest of these three levels is the visceral, which responds to sensory stimuli and initiates motor behavior. The second level is behavioral, which also responds to stimuli and can initiate behavior. The behavioral level can be influenced by the reflective level, whereas the visceral cannot. The highest level is the reflective level, which is isolated from the sensorimotor actuators. It monitors the behavioral and visceral levels and influences the behavior. 
Irrespective of the cognitive processing and the meaning attached to an object or event, it is the emotional response that assigns value to that object or event. Paraphrasing Norman (2004), a user, after consideration of all the "facts," may still respond, "It does the job but it's the wrong color: I don't like it," and that is the final decision. Therefore, in considering system acceptance and the UX, for the research reported in this paper, the user's emotional response is always given higher precedence over the reflective level.

\section{Affordance in Intuitive Use Design}

The move from the command line on a blank screen to a menu on the graphical user interface changed the mode of interaction between the user and the computer. A graphical user interface application incorporates windows, icons, menus, and pointers for the user to monitor and control the interaction, and thus what-you-see-is-what-you-get elements present the content. These screen attributes are important when considering intuition and future and continued use: Screen layout based on artifact selection and location is fundamental to the direct manipulation paradigm for interaction design.

The term affordance (Gaver, 1991; Hartson, 2003; Norman, 1988) refers to the cognitive (perceived) and physical (actual) properties of an artifact. Artifact affordances encapsulated in the physical properties of an object, such as its size, shape, color, component materials, location, and so on, are visually discernible and provide the observer with clues as to the object's nature and purpose. By design, then, affordances should provide strong visual clues to the use possibilities and to the operation of the artifact so that, on inspection, the user knows what it is used for and how to use it.

In practice with new artifacts, users typically are unfamiliar at first with the visual representation of the related commands and the methods to apply those commands in the direct manipulation interaction paradigm. Consequently, large discrepancies can take place between what inexperienced users expect to occur and the actual outcome of their actions. To counter the problem of misinterpreting screen artifact affordances, the artifacts usually are made to visually resemble the real-world object in form and affordances, and they operate in a similar manner as in the real world. Similarly, objects encountered only on the screen are made visually and operationally consistent over platforms.

The need to develop these attributes in screen artifacts is explained by distributed cognition (Hutchins, 1995, 2000). Distributed cognition moves beyond the individual to the system, identifies the mechanisms by which the individual shares within the praxis of a community and how tools propagate that praxis across the members of the community over time, and then subsequently provides the user with what is already known. Distributed cognition adheres to the principles that the use practice and associated cognitive processes are

- distributed across the members of a community and consequently across locations,

- distributed between the cultural artifacts and tools, and

- distributed across time.

In applying these principles, the intent of design should be to use as much of the users' expertise as possible, and where that is not possible, to align the new activities as closely as practical with existing tools and practices. In this context, design is not a clinical analysis, but rather the interaction with use experts to extract, understand, and extend existing practice. 
This means that the artifacts and their cognitive and physical affordances, including their location on the screen, should be drawn from the praxis of the largest subset of users in the community of practice. To design with this in mind means that the majority of users are already familiar with the artifacts and how to use them (Lehane \& Huf, 2006, 2007). By design then, the activity praxis is distributed over time, location, and people: This leveraging of prior knowledge is a contextual example of distributed cognition. From the developers' perspective, the cliché used in requirements analysis to implement this concept is "make it intuitive." For this article, which ascribes to the concept of scripted behavior, the definition of intuitive is to "give the users what they already know."

Consideration of the community of practice was used to examine both the crystallized practice in the artifacts and the means by which the practice was both crystallized and propagated (Kuutti, 1996). Good interaction design based on distributed cognition/user-community praxis should place the appropriate artifact in the right place on the screen most appropriate for its use and at the right stage in the sequence of actions for that activity. The mantra "Know your user" should be considered a condensation of the broader and more fundamental concept from distributed cognition, which is "Know your users' community of practice."

This concludes the pragmatic HCI-focused discussion on how intuitive design as scripted behavior drawn from exemplar communities of practice (i.e., Mac users vs. PC users) is implemented in the interpretation of the responses to the SAI. The concept of scripted behavior has been well-documented in the discipline of HCI. Scripts have been used in the discussion on how experienced personnel, such as firefighters, plant operators, and air controllers, analyze and respond to known and, in particular, unfamiliar situations (Bainbridge, 1997; Jones, Chu, \& Mitchell, 1995; Kontogiannais, 1996; Pawlak \& Vicente, 1996). In this case study, I am explicit in stating that the premise of scripts was fundamental for the development of the theoretical rationale used in the discussion to explain the observed user behavior.

\section{INTERACTION AND SYSTEM DESIGN CONCEPTS}

In this section, I discuss the inclusion of functionality in the usability engineering model (Nielsen, 1993) so that concepts from soft systems modeling could be used to construct a theoretical rationale for the emergent construct of the usability engineering criteria. Similarly, the HCI concepts used to consider the inclusion of intuitive criteria in design, along with other HCI concepts that were used to classify the observations and data collected from users, are assembled to operationalize activity theory. Finally, these concepts are put together into a framework that provides a design rationale and checklist for implementing the praxis of a community of practice.

\section{Usability in System Design}

Throughout the 1990s, one design paradigm underpinned all others: usability (Carroll, 1991; Nielsen, 1993; Preece, Rogers, \& Sharp, 2002, 2007; Preece et al., 1994). Nielsen (1993) defined the system acceptance attributes. For a system to be accepted, it had to be socially and practically acceptable. System performance considered the mechanical attributes of the system and whether the system was fit for its intended purpose. Nielsen addressed system performance by assessing the usefulness, functionality, utility, and usability of the system. Functionality is a key criterion 
for requirements and gap analysis, and for the design of industrial/commercial systems, but functionality was not represented in the graphical representation of Nielsen's published model (1993, p. 24).

To relate a grounded theory analysis of the survey responses to the commercial domain of mandatory-use systems for the research project on which this article is based, it was necessary to develop a theoretical relationship between functionality and the other usability engineering criteria. Both utility and usability are, by definition, dependent on and qualifying attributes of functionality, drawing on the human factors definitions of tasks being assigned to humans and functions allocated to machines (Stammers \& Shepherd, 1995). Functionality, thus, was deemed to be how well the system could support each of the individual functions allocated to it in the breakdown of the work. This aligned with the users' interpretation of system functionality being the functions programmed into the system and the requirement to assemble functions into action sequences for business processes (Lehane, 2012b). Therefore, functionality had to be a node between usefulness and the functionality qualifiers of utility and usability. A modified Nielsen model of the criteria determining system acceptance that includes functionality and emotional acceptability (Norman, 2004) is presented in Figure 1.

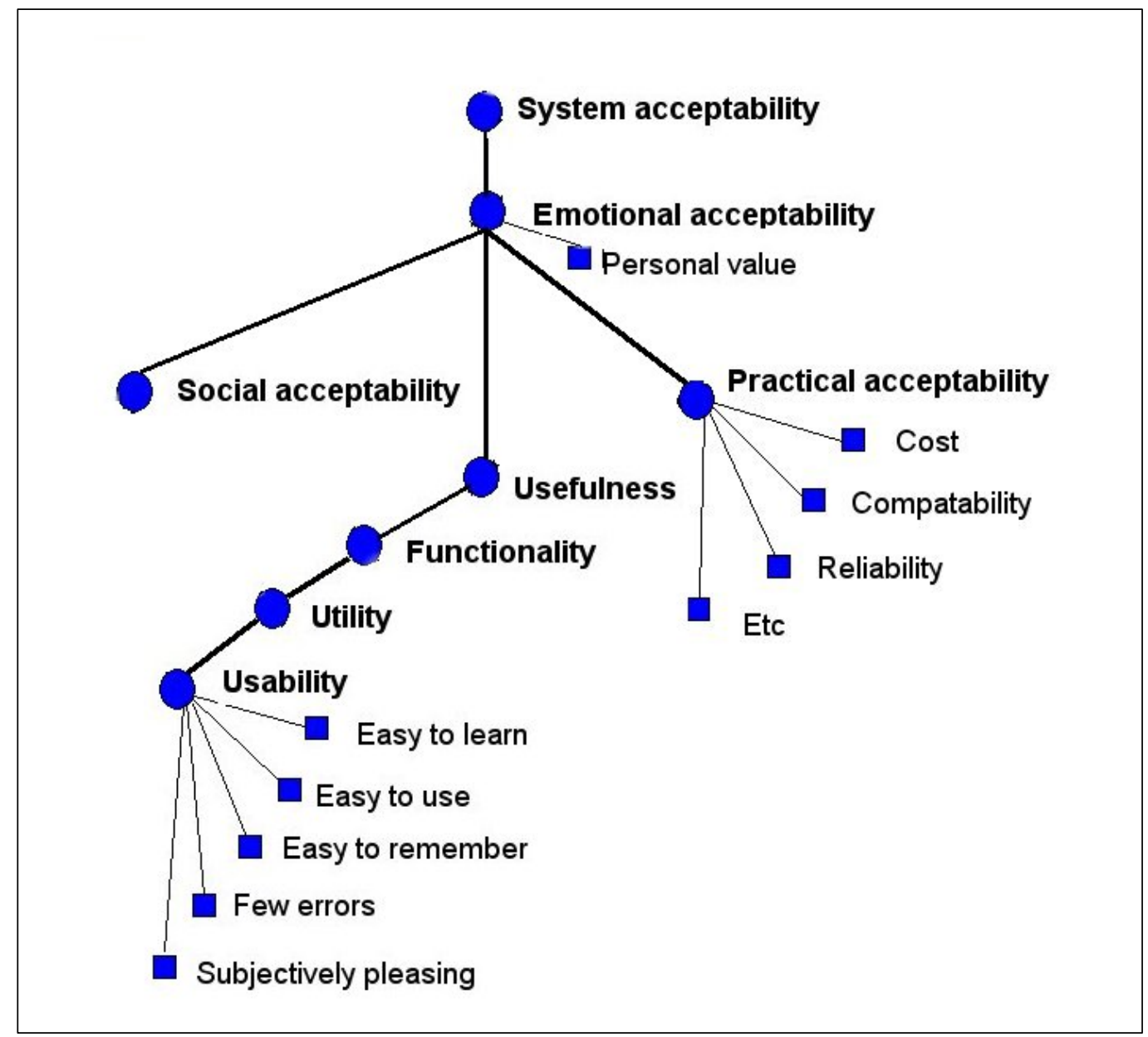

Figure 1. Functionality as a node between usefulness and utility in a system acceptability model. 
The five attributes of usability relate as well to requirements for induction into a community of practice: the learning experience in general and the operationalization of an action and reflective appraisal on usefulness (Lehane \& Huf, 2005). From this perspective, the system acceptance attributes of usefulness, functionality, utility, and usability are usability engineering criteria that can be used to assess the effect of technological change on the cognitive UX (Lehane, 2008).

\section{Abstraction Hierarchy for Structured Interaction Design}

From a theoretical perspective, systems engineering was founded on two pairs of concepts: hierarchy and emergence, and communication and control (Checkland, 1999). Hierarchy addresses concerns with the fundamental differences between one level of complexity and another. It provides both an account of the relationships between the different levels and an account of how observed hierarchies come to be formed: What generated the levels, what separated them, what linked them? Emergence at one level in a hierarchy is associated with constraints upon the emergent elements. The emergent properties are meaningless within and cannot be communicated by the language appropriate to the lower level. This imposition of constraints upon activity at one level is a control action that harnesses the laws at that level to yield activity meaningful at the higher level.

Checkland (1999) illustrated emergence with the example of the genetic code within the structure of DNA, where the genetic code was an emergent property from chemistry and marked the transition between chemistry and biology. As such, DNA structured by the constraints of chemistry on the genetic code characterized biology. In a similar manner, the usability engineering properties mark the transitions in the emergent hierarchical structure of the interactions that characterize but do not define system acceptance.

For the research reported in this article, the soft systems modeling theoretical concepts of hierarchy and emergence and communication and control were applied to assemble theoretically an abstraction hierarchy for usability engineering. This theoretical hierarchy defines the relationship between artifact and interaction. It also facilitates the classification of the screen artifacts within the structure of the usability engineering. In so doing, the abstraction hierarchy links the artifacts to the interaction and the interaction to activity-system acceptance. The establishment of these relationships between artifact, interaction, and system acceptance was necessary for the theoretical integrity in explaining/justifying the interaction model that validated the grounded theory analysis-derived classification of the emergent UX characteristics.

Returning to the implications of Checkland's (1999) genetic code exemplar interpreted for the parallel realities discovered in the interaction model, an instance of emergence is artifact affordance, which is emergent from the constraints imposed on artifact design by use. The artifact's affordances impose constraints that structure the artifact's usability and characterize its interactions. In other words, the only interaction possibilities are those provided by the affordances of the artifacts. Affordances, as the vehicle of distributed cognition, are the use community's praxis crystallized in the artifacts; they transport the history of use across time, location, and the population of the community of practice. These constraints produce the specific actions and scripts for the usability of an artifact and characterize the interactions. Affordances mark the transition from the level called artifact to the level called interaction, as depicted in Figure 2. 


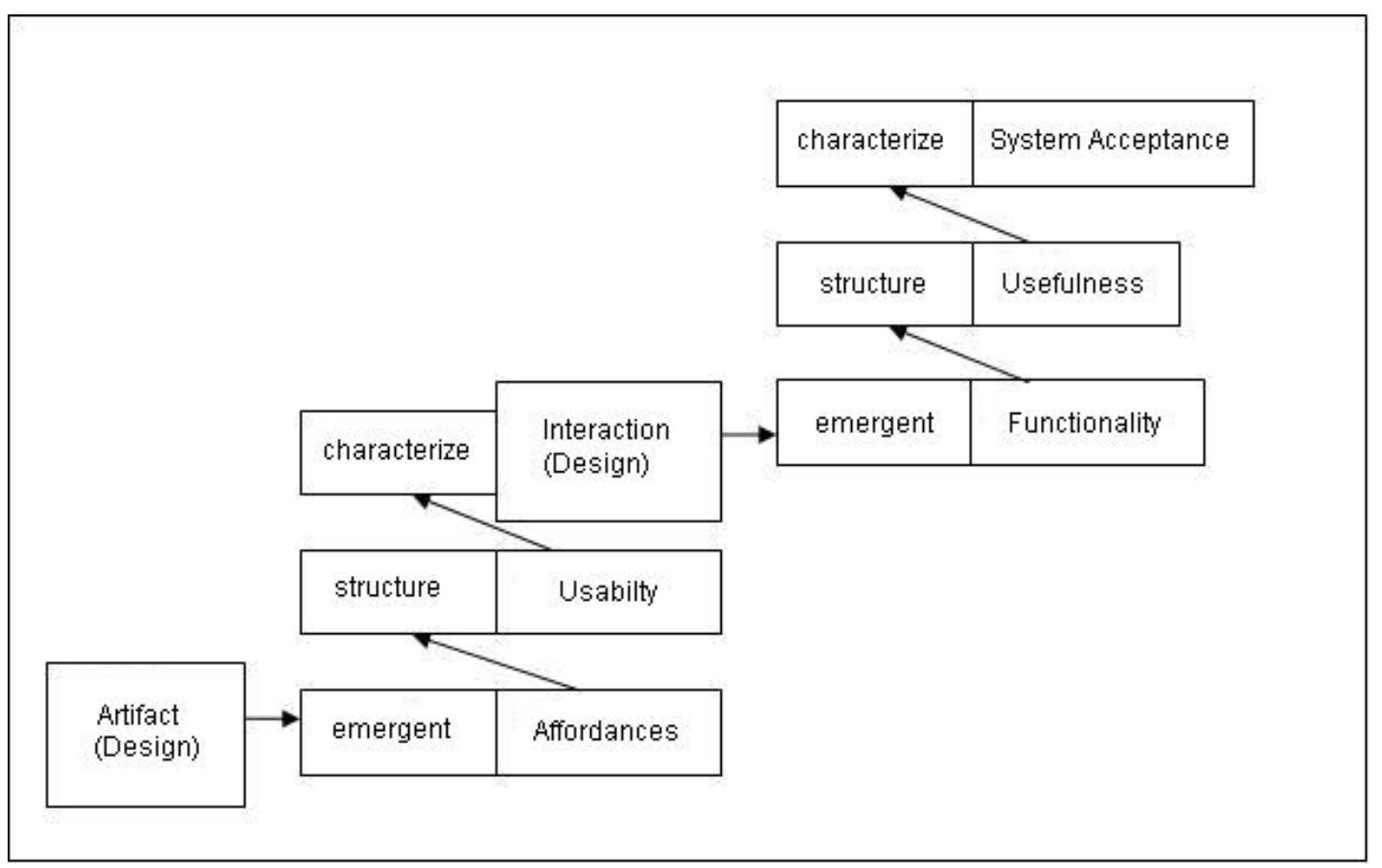

Figure 2. Emergence abstraction hierarchy for usability engineering.

At each stage of any human-computer interaction, myriad operational options exist. But to successfully complete the activity, the sequence selected from the available actions is critical and that sequence constitutes the interaction and the realization of a situated plan. For a system to be accepted, the functionality built into the system by interaction design must support all the tasks assigned to the human by workplace task allocation, as well as all the functions allocated to the machine for the operation of the system. To deliver the required business/operational capabilities as outcomes, the functionality must be perceived to support the tasks in a useful manner, thereby positively influencing the affective systems of the user. If all the tasks are not supported, the usefulness of the system is compromised.

System acceptance, as depicted in Figure 2, is an emergent level characterized by the usefulness of the system. Usefulness structured by the functionality is constrained by an interaction design based on artifact-use possibilities. These interaction design constraints result in the activities supported by the functionality, an emergent property that marks the transition from the level called interaction (with the system) to the level of system acceptance. Functionality embodies the expert-user praxis built into the system by interaction design. As such, it constitutes the functions allocated to the computer to support the tasks assigned to the human as purposeful activities.

Functionality constrained by interaction design provides a structure for the emergence of usefulness, which characterizes but does not define system acceptance. Well-structured usability, based on artifact affordances, is required to ensure comprehensive interaction design so that the user will interact usefully with the functionality for system acceptance. The artifact-interaction transition is an operational state change negotiated between the object and the activity. The interaction-system acceptance transition is an affective-behavioral-reflective system state change negotiated between the activity and the motive for the activity, which is 
why usefulness characterizes but does not define system acceptance. The trisystem response is not controlled solely by the situated usefulness considerations of efficacy, efficiency, effectiveness, ethics, and elegance (Checkland, 1999); it also is influenced by the value the user attributes to the meaningful outcome of the interaction (Norman, 2004).

System acceptance, by definition, is a measure of the system's ability to satisfy all the needs and requirements of all the users and potential stakeholders (Nielsen, 1993, p. 24). Two things need to happen for system acceptance. First, the usability of the artifact's affordances, constrained by artifact's design in a series of interactions at the action level, has to produce an activity system wherein the usefulness of the functionality, constrained by the interaction design, facilitates the user in attaining an objective. The second and more important thing that needs to happen is that the experience within the cognitive meaning of the task influences the user's affective system to apply value to the activity.

The UX is the internalization of these affective, behavioral, and cognitive experiences. The emergent subjective assessment is an overarching experience that is integral to the theme of this article. In this context, a fit for purpose and a value-adding experience are both required for a system to be considered useful, leading possibly to user acceptance.

\section{Activity Theory and Interaction Design}

This article proposes activity theory as a capstone theory to explain the phenomenon observed and studied but does not always look to activity theory to investigate each of the individual activities. Activity theory considers consciousness - as well as the asymmetrical relation between people and things - and the role of artifacts in everyday life (Nardi, 1996a). Consequently, information processing and information systems should not be seen as something to be modeled in the same way for both people and machines. The activity of an individual is part of the collective activity of various groups. Within these groups, each specific activity consists of communication with others to organize, coordinate, and control the actions directed towards the artifacts and facilitate the activity (Kuutti, 1996). The coordination and organization are called the communicative side of human activity, and the control directed towards the artifacts is called the instrumental side.

The communicative side of human activity makes use of a rich array of linguistic, nonverbal, and inferential resources to understand actions and events. However, on the instrumental side, machines rely on a fixed array of sensory inputs, mapped to a predetermined set of internal states and responses. The result is an asymmetry that substantially limits the scope of possible interactions between humans and computers (Kaptelinin, 1996).

Consequently, when problems arise, they cannot be resolved from the instrumental side unless the issue was previously considered in the design and the resources to re-establish or maintain the interaction are already available. However, the operator has, from experience or training, a repertoire of scripts and action sequences with which to attempt to reopen or maintain the interaction. When the interaction is re-established and can be successfully concluded, a workaround has been established from the communicative side. Subsequently, user practices should confirm the explicitly supported activities, and expose the tacitly supported activities, which will require task-specific functionality in the interaction design. 


\section{Activity Theory Operationalized}

Activity theory does not accept the duality of an isolated and independent mind; the internal side of an activity cannot exist without the external in activity theory (Bødker, 1991; Hutchins, 1995; Nardi, 1996a; Suchman, 1987; Vygotsky, 1978). Typically, before an action is performed in the real world, it is consciously planned using a model. The term for this is orientation: the representative of the internalization of the motive for the activity. Orientation directs the individual's assessment of the activity, linking the internalization of the motive and the formulation of a response structured by the external influences of experience and environment.

Engeström (cited in Kuutti, 1996) extended Vygotsky's element-mediated relationship of tool, subject, and object to include the relationships between rules, community, and division of labor. To represent the theoretical base of this article, additional operational detail was incorporated into the Engeström diagram (see Figure 3). This was achieved by positioning the individual's response in the model as an orientation activity between object and outcome: Orientation locates the subject's consciousness as the affective, cognitive, and physical responses with and in the context of the object-outcome transformation process (Checkland, 1999; Norman, 2004; Preece et al., 2007; Shiizuka, 2007).

With this extension, the contingent, responsive, and improvisatory emphases of situated action and the conscious human motives and systemic goals of activity theory and distributed cognitions are included in the model. Adding the orientation activity to the transformation process of the model brought together the two activity theory themes of context and consciousness (Nardi, 1996b) and made them explicit in the representation. The iterative cycle was placed around orientation to indicate that the realization of the motive and the synthesis of the means to achieve an outcome are not linear processes.

As illustrated in Figure 3, activity theory was used to provide the rationales for both the evaluation of a community of practice and an activity. Cognitive HCI research into artifact design and development has provided models and theories to explain artifact selection and use. The conceptual intent of the action research project was to investigate the individual activities and describe them using the original philosophical and cognitive HCI paradigms under which they were first examined: HCI concepts such as the active user (Carroll \& Rosson, 1987), which was the means to consider the individual in the greater use community; affordance (Norman, 1988) as the mechanism for imparting practice into tools and artifacts; direct manipulation (Shneiderman, 1982,1983 ) for the protocols to physically engage with and use the tools and artifacts; distributed cognition (Hutchins, 1995, 2000) as the means for propagating that praxis over time, location, objects, and communities of practice; and soft systems modeling (Checkland, 1999; Checkland \& Holwell, 1998) as the mechanism to establish tool-use rules for the community and thereby individuals, and consequently the means to conceptualize between the object and the outcome. Scientific management (Taylor, 1911) provided the rationale for the division of labor within the community of practice, and situated action (Suchman, 1987) explained purposeful activity as the contextually determined implementation of the object as an outcome.

One HCI paradigm does not clearly and solely relate to each of the elements in the extended activity theory model; they overlap. The HCI paradigm that was perceived to be dominant in this research was placed at the top of the list and the others were placed by order of perceived influence. The concepts presented in this article were incorporated into the original action research project to establish its theoretical framework for interpreting the user response to 


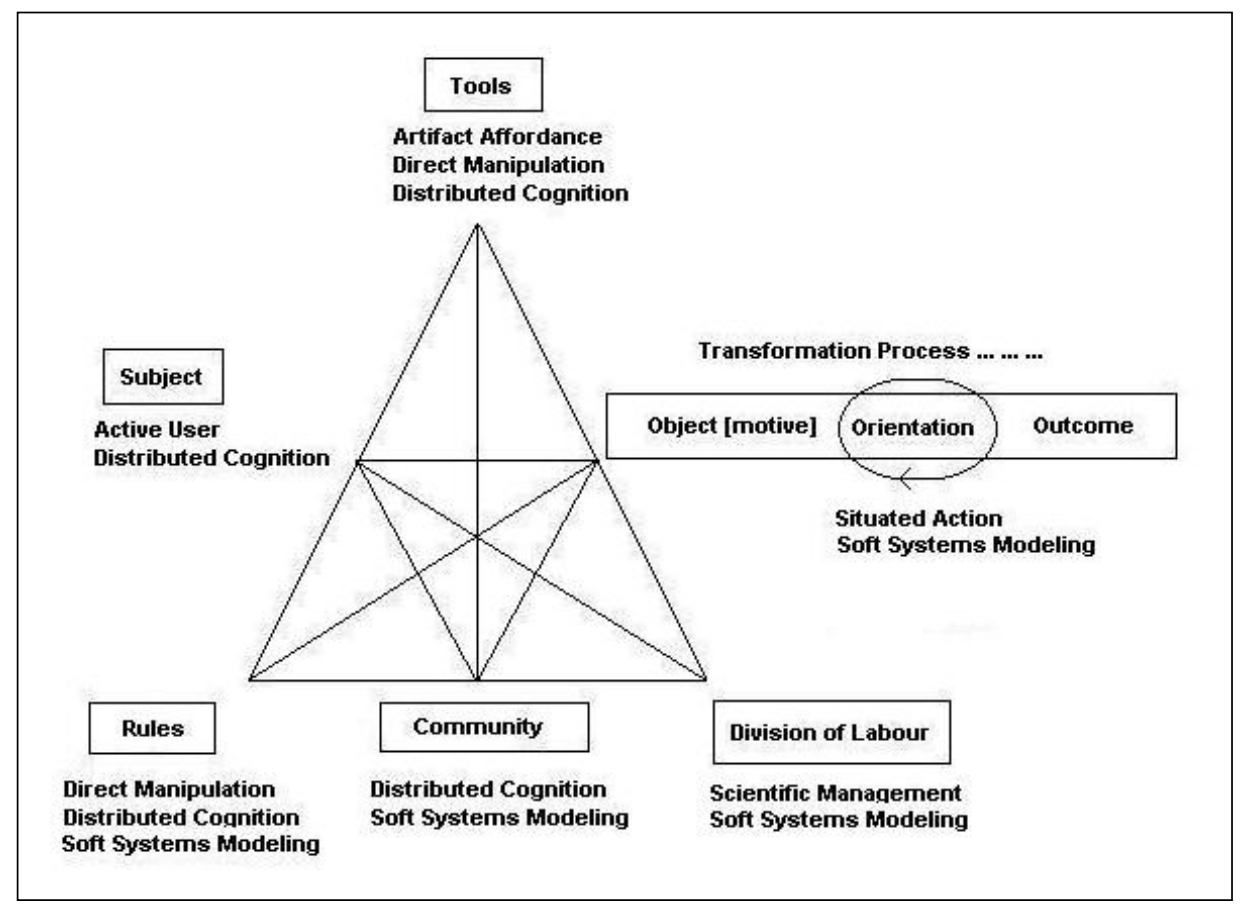

Figure 3. Concepts from cognitive HCI to operationalize Engeström's activity theory model.

the SAI. The project's pragmatic interpretation of activity theory showed, in this instance, that by subsuming these models and theories of cognitive HCI, activity theory provides an encompassing theoretical basis for explaining the complexities of HCI and, conversely, these models and theories of cognitive HCI are a plausible methodology for operationalizing activity theory.

\section{Community of Practice Interaction Framework}

This section distills the essence of my interpretation of the HCI models and theories as presented in this article and used in an operational context in the case studies of earlier publications (Lehane, 2008, 2012b; Lehane \& Huf, 2006, 2007). Humans mediate their activities with tools. The introduction of technology to mediate human activity is a learning process that markedly reshapes the nature of the activity during the period that the artifact transitions to a tool. To understand the present use of a tool, it is necessary to understand the way the tool's use and design change over time. The community focus for tool use is on ease of learning, ease of use, and usefulness within the use goals and context. The development of the tool arises out of these needs. It is important to note that a tool can be a physical object, such as a computer, or a concept, such as intuitive design.

In design, the desire or design objective to make the use of a tool intuitive can be achieved by drawing on previous experience and building on the knowledge the intended users already possess. The experiences on which the design is based must originate from the practice of the largest subgroup of the use community and be nearly universal within that community. If the experiences are familiar to only a small subset of the use community, the practice will have to be learned by the extended community. The accretion of knowledge and the acquisition of new skills are then crucial to acceptance. This learning experience is shaped by the culture of the 
community and the learning process itself. In this context, the use of the tool is made explicit by its affordances. The computer screen, by way of the layout and artifact design, establishes the terrain for the interaction and contains the ecological attributes and contextual constraints necessary to realize the objective as a computer-mediated activity. The interaction framework derived from consideration of these concepts is presented in Figure 4.

The models and theories of HCI as structured in Figure 4 are about interpretation and learning. They embody the distinction between the optimization paradigm of hard systems thinking and the learning paradigm of soft systems thinking (Checkland, 1999). In hard systems thinking, the social world is assumed to consist of systems whose performance can in some sense be optimized. Soft systems thinking, and the soft systems modeling that embodies it, assumes a more fluid social world, one that both persists and changes. This means that, in accord with Checkland and Holwell (1998), this interaction design model seeks interpretation and learning rather than optimization.

The model begins with an individual or group interested in learning to use an application. As an active user, the individual or group initiates interest in the application and its use. The active user is proactive in gaining familiarity with the application. This endeavor involves self-learning and assisted learning. The learning materials and trainers come from the community of practice. The training provides familiarity with the praxis of the community of practice. The praxis integrates not only the use practice, but also the history, the myths, the champions, the artifacts, and the culture of the community. These constitute the distributed cognition of the community. In the ICT domain, the distributed cognition encompasses the screen layouts, the design of the screen artifacts, the users' expectations for interactions with the icons and menus, and the expected machine responses from user interactions with the screen. The use practice necessary to produce

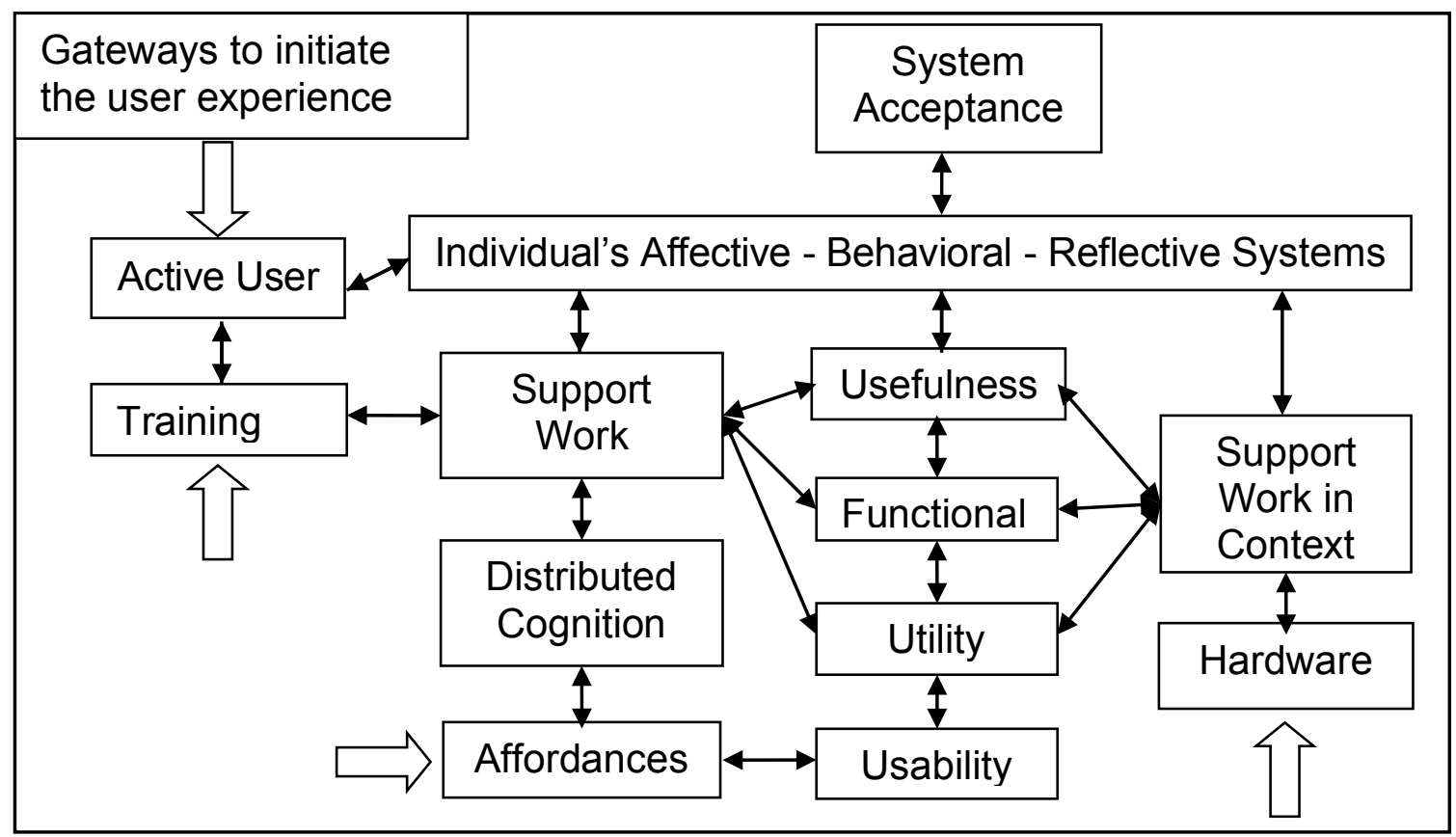

Figure 4. Computer community of practice interaction model. 
the desired outcomes is intrinsic to the artifacts' physical properties, which are shaped by use and use possibilities.

The community of practice has measures for artifact performance that are used to determine if the tool is fit for purpose and acceptable; concept-wise they are usability, utility, functionality, and usefulness. Usability relates directly to the use of the physical objects (or concepts) and their affordances. Utility is the efficiency of the use actions, while functionality is the scope of the use activities. Usefulness is the fit-for-purpose reflective assessment. These integrated contextual activity concepts address the use of the artifacts with respect to the quality of the interaction and the outcome.

The system typically is considered usable when all these elements come together so that the user gains enough familiarity with the application to use it and to produce the desired results. In addition, if the experience of using the application to achieve the desired results evokes a positive emotional response, the system may be accepted by the user and used again. But irrespective of the system producing the desired outcome, should the experience evoke a negative emotional response, it most likely will not be repeated. This negative outcome holds especially true in voluntary-use situations. However, in mandatory-use situations, acceptance is independent of the end user's emotional response and is determined alone by management's perspective on usefulness. Nielsen's (1993) criterion of practical acceptability would have been established by the management decision to purchase the system.

\section{THE SURVEY AND GRAPHS}

Before discussing the statistical analysis, it is necessary to introduce the basic concepts behind the survey and the graphical presentation of the users' responses. More detail and use instances from case studies are available in previous publications specifically for this purpose (Lehane, 2012a, 2012b). The SAI (Lehane \& Huf, 2005, 2006) survey contains 25 questions about the positive and negative aspects of system use. Each question is assigned a value from 0 to 4 , with 4 representing strongly agree with a positive aspect of use in the odd-numbered questions but strongly agree with a negative aspect of use in even-numbered questions. (See Lehane, 2012b for a fuller description.) Even-numbered questions were adjusted so to allow for a perfect score of 100. The global index for one survey is the summation of the values assigned to the response to each question; the SAI global index for a survey campaign is the average of the individual indices. This is similar to the way that the system usability scale (Brooke, 1986) works: The questions were grouped and the responses averaged to create graphs during the analysis.

The SAI provides three measures. The first element is a global index as a number between 0 and 100. Fifty is the value of the global index indicating a neutral disposition towards the system. Zero indicates a system that is perceived unfavorable for all questions and 100 is the score for a system that received the maximum of favorable responses.

The second measure is the graph for the data determined by the technology acceptance model (TAM; Lehane, 2012a; Lehane \& Huf, 2005, 2006), which is a 12-element presentation of the users' perceptions of the system. An example of this graph follows in Figure 5. The criteria for this graph are expressed in analytical terms for technical consideration of the results by the system developers. 


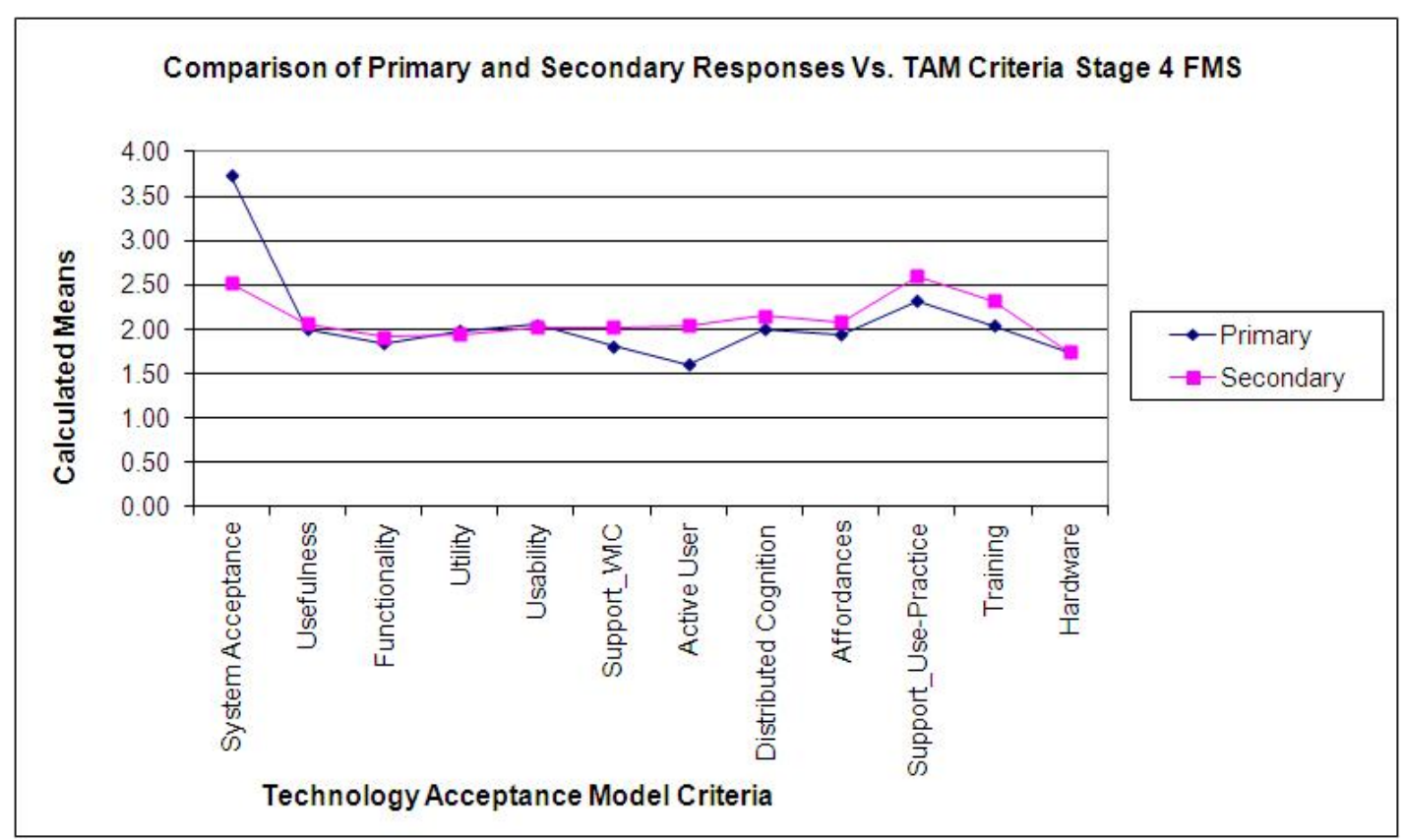

Figure 5. A graph of the technology acceptance model.

The five criteria from usability engineering describe immediate use:

- System acceptance is how well the users relate positively to the system.

- Usefulness is how well the overall system supports users in achieving their objective(s).

- Functionality is how well the system's functions support the designed activities.

- Utility is how efficient the system is in facilitating the actions.

- Usability is how effectively the actions can be operationalized.

The seven concepts identified as the use-community criteria compare the use of system with previous use knowledge and experience:

- Support for Work-in-Context (Support_WIC) is how well the system integrates into the extant workplace systems.

- Active User is the level of proactive interaction initiated by the user.

- Distributed Cognition is how well the praxis of the domain's community of practice was transferred to the software (i.e., does it have a familiar look and feel?).

- Affordance is how well the context of that praxis was embedded in the artifacts (i.e., was use intuitive?).

- Support for Use-Practice (Support Use-Practice) is the level of immersion of the user into the community of practice (e.g., an accounting background for a finance officer ensures comprehensive contextual knowledge).

- Training is the formal training and its cognitive and behavioral artifacts used to transfer use-practice from experts to novices.

- Hardware is concerned with issues related to the situated technology (i.e., computers and network). 
The third measure is the SAI graph, wherein the survey responses are regrouped to a 10element graphic presentation of the users' experience in nontechnical terms. The SAI graph is used as the basis for discussions with the business users of the system being surveyed. Figure 6 is a graph from the case study of a financial management system (see Lehane, 2012b). The TAM technical criteria of active user and distributed cognition are grouped in the SAI graph as EZ2Learn, while affordances and support for use practice are combined as EZ2Use. EZ2Learn is an indication of the active user's ability to leverage prior knowledge through the use of distributed cognition. EZ2Use is an indication of the affordances and use community praxis facilitating recall and operationalization of activities. Conceptually these two categories are associated with and provide an indication of the "look and feel" of the system and how intuitive the software is to use.

The adjusted individual survey responses are collated to compile the collective response to the UX. The survey ratings scale of 0 to 4 thus covers the range of the collective response from strongly negative to strongly positive. The guide for interpreting the scale is

- 0 - total rejection

- 1 -poor response $<1.5$ indicates a criterion to be looked at

- 2 - normal expectation, no significant influence

- 3 -good response $>2.5$ indicates a criterion that was well received

- 4 - full acceptance.

The SAI was designed to provide a global indication of user satisfaction and identify the users' rationales for reaching that decision.

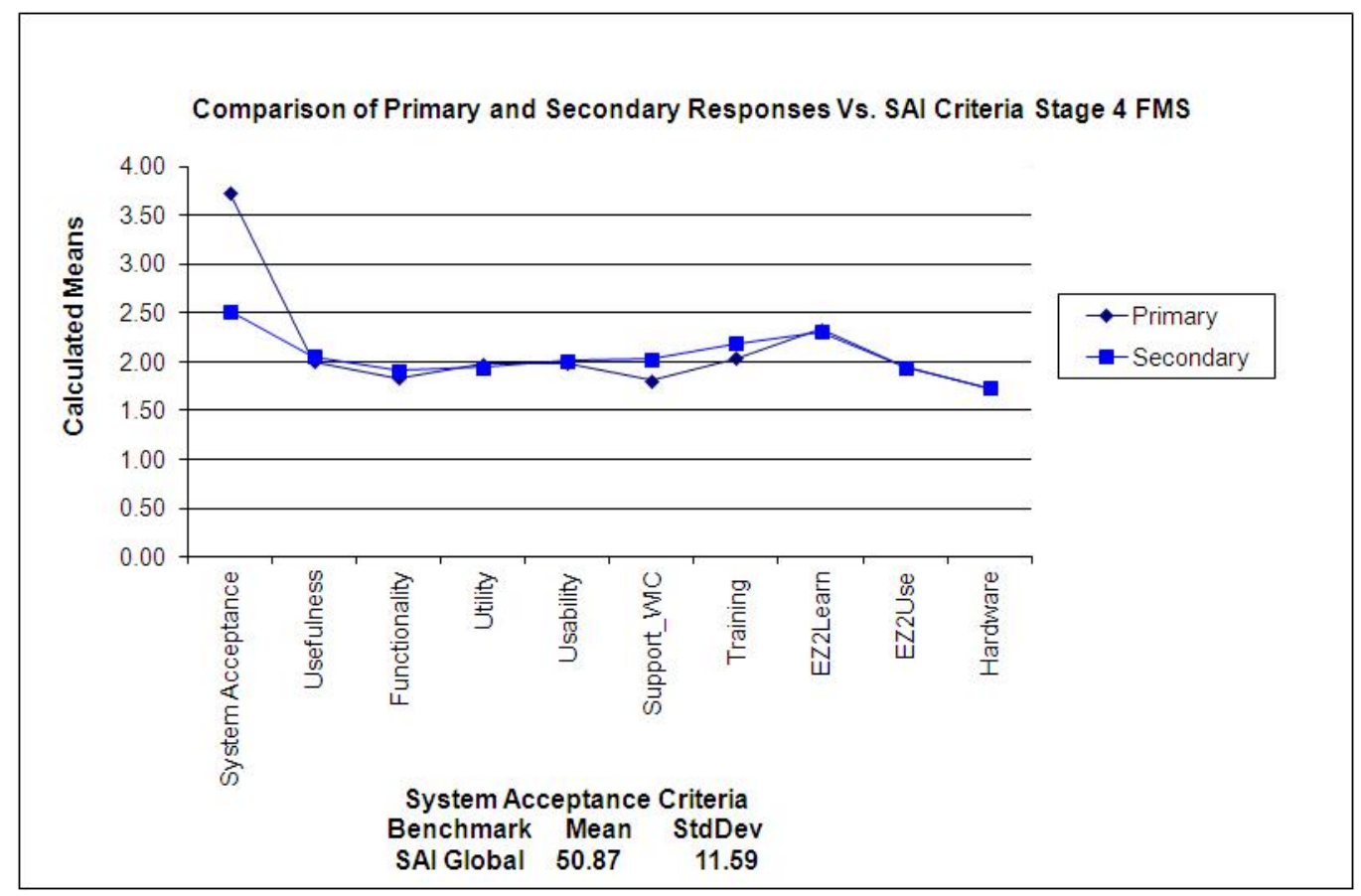

Figure 6. A graph of the System Acceptance Indicator. 


\section{STATISTICAL ANALYSIS RESULTS}

The SAI data underwent statistical analysis and the details are provided in Lehane (2012a); only the results are presented here. The survey is the data-driven emergent theoretical questioning that is integral to the grounded theory analysis. A tenet of grounded theory is that the data and associated theory emerge conjointly and dependently upon each other. In Table 1, the emergent structure from the data analysis closely aligns with the grounded theory-emergent theoretical structure. This confirms the methodology, wherein the analysis is driven by the data in such a manner that the final theoretical constructs are a good fit to the research context.

Principal component analysis of the data $(N=376)$ identified a Component 1 that accounted for $41 \%$ of the variance in the user responses. The variable Usefulness was the greatest contributor to Component 1 . The variables of Component 1 were elements from the abstract concept of usefulness (fit for purpose in situated use), and the effectiveness and efficiency of the system being used. Because of this consistent theme in the variables of Component 1, it was called Situated Use.

The analysis also identified a Component 2 that accounted for $28 \%$ of the variance. The variable EZ2Learn was the greatest contributor to Component 2. The variables in Component 2 carried the theme of familiarity in acquisition and implementation of use-practice. A theme in this article is intuitive or scripted behavior, which was interpreted as giving the users what they already know. For this reason and the variables concerned, Component 2 was called Intuitive Use.

The structure of the variables in Table 1 follows that of an abstraction hierarchy emergent from the user description of their interactions with computers. Usefulness is the highest and most abstract reflective concept. The relationship between the usability engineering and user community criteria is that the usability engineering criteria present the response to current use and interaction with a system, and the user community criteria present the use of knowledge from previous computer-use experience. The user community criteria relate to relationships between the system and the work. The conceptual requirements of usability are easy to learn

Table 1. Data Analysis Supports the Abstraction Hierarchy of the Emergent Theoretical Construct.

\begin{tabular}{cccc}
\hline $\begin{array}{c}\text { Technology Acceptance } \\
\text { Model Reflective Criteria }\end{array}$ & $\begin{array}{c}\text { Reflective Elements in } \\
\text { Key Concepts }\end{array}$ & $\begin{array}{c}\text { Reflective Elements in } \\
\text { Component 1 } \\
\text { Situated Use }\end{array}$ & $\begin{array}{c}\text { Reflective Elements } \\
\text { in Component 2 } \\
\text { Intuitive Use }\end{array}$ \\
\hline $\begin{array}{c}\text { Usability Engineering } \\
\text { Criteria }\end{array}$ & Usefulness & .781 & \\
& Functionality & .749 & \\
Ustility & Usability & .736 & .815 \\
Use Community Criteria & Distributed Cognition & & EZ2Learn \\
& Active User & & .949 \\
& Support Use Practice & EZ2Use & \\
Ecological Criteria & Affordances & .734 & \\
& Support Work in & .695 & \\
\hline
\end{tabular}


and easy to use: These concepts are presented in the user community criteria as the characteristics EZ2Use and EZ2Learn.

The component Situated Use comprises variables from all three criteria classes. The contribution of the variables to the component reveals the influence of reflection in use assessment. The structure of this component indicates the importance of the high-level abstract concepts of usability engineering in shaping the user's response to system use, with usefulness emergent as the dominant consideration in system acceptance. The component Intuitive Use indicates the importance of usability as a secondary consideration. If the usability is such that it allows a user to gain enough familiarity with the system so as to be able to determine its usefulness, then, upon reflection, an evaluation may consider the system fit for purpose.

The development of the theoretical structure was integral to the development of the criteria, as they both evolved with the grounded theory analysis and sampling. The presentation of the abstraction hierarchy for the system acceptance criteria beside the emergent experimental data structure clearly shows the relationship between the theoretical constructs and research findings.

\section{CONCLUSION}

In this article I discussed the development of the theoretical rationale for classification criteria for the SAI survey, which came from applying concepts from a grounded theory analysis to users' responses to surveys and focus group interviews. Grouping the users' responses to the interviews and surveys ensured consistent analysis and interpretation of analysis results. The users described the system requirements and the problems they encountered, and from those descriptions the survey criteria surfaced classifications that grouped the issues.

Once classified, a number of concepts from HCI paradigms could be applied to specific issues in the users' descriptions of their problems and their requirements. In addition, this work indicated that it might be possible to use the HCI concepts to develop a structured theoretical rationale to support the recommendations made using the notes and analysis coding system. Consequently, concepts from a number of paradigms were brought together to form the contextual interpretation rationale for the survey presented in this article.

A grounded theory analysis ensures that the research data is theoretically accountable. The survey campaigns presented a large sample of numerical data on the software upgrades and the associated UX (see Lehane, 2012b). Those data were used for statistical analysis to assess and confirm the emergent theoretical construct of the usability engineering criteria and the user community criteria. The statistical analysis validates the SAI survey.

Usefulness, a characteristic of the theoretical constructs of this research, was emergent as the dominant variable in the principle component analysis of the research data. In addition, data dependent on and emergent from the component variables supported the abstraction hierarchy, which mirrored the proposed theoretical interaction hierarchy. The emergence of usefulness, the most abstract variable, as the greatest contributor to the component Situated Use is significant. It is seen to endorse the soundness of the data-determined theoretical model developed and used in this project.

When a series of surveys is taken over a period of time at critically specific transition points, the subsequent graphs present the UX in HCI terms. The strength of the SAI, a 
validated survey instrument, is that its methodology incorporates the analysis interpretation and an issue mitigation premise; both are based on HCI models and theories.

Finally, the analysis methodology utilizing community of practice concepts has been used successfully in various applications to resolve real-world issues associated with software upgrades. The central theme for the analysis interpretation and issue resolution was communityof-practice scripted user behavior, based on the tool-associated practices of the use community. This HCI convention of leverage of prior knowledge was implemented as "give the users what they already know" and interpreted as intuitive design. Because the survey identified user concerns at a high level of abstraction and also presented a means to rectify those issues, the survey and the premise behind it were seen to be validated in practice.

\section{REFERENCES}

Bainbridge, L. (1997). The change in concepts needed to account for human behaviour in complex dynamic tasks. IEEE Transactions on Systems, Man, and Cybernetics, 27(3), 351-359.

Bødker, S. (1991). Through the interface: A human activity approach to user interface design. Hillsdale, NJ, USA: Lawrence Erlbaum Associates.

Brooke, J. (1986). System usability scale. Reading, England: Digital Equipment Corporation.

Carroll, J. (Ed.). (1991). Designing interaction: Psychology at the human-computer interface. New York, NY, USA: Cambridge University Press.

Carroll, J., \& Rosson, M. (1987). Paradox of the active user. In J. M. Carroll (Ed.), Interfacing thought: Cognitive aspects of human-computer interaction (pp. 80-111). Cambridge, MA, USA: MIT Press.

Checkland, P. (1999). Systems thinking, systems practice: Includes a 30-year retrospective. Chichester, England: John Wiley and Sons.

Checkland, P., \& Holwell, S. (1998). Information, systems and information systems: Making sense of the field. Chichester, England: John Wiley and Sons.

Dick, R. (2005). Grounded theory: A thumbnail sketch. Retrieved August 2, 2006, from http://www.aral.com.au/resources/grounded.html

Gaver, W. (1991). Technology affordances. In M. Altom \& M. Williams (Eds.), Proceedings of CHI'91 (pp. 79-84). New York, NY, USA: ACM.

Glaser, B. (1994). More grounded theory methodology: A reader. Mill Valley, CA, USA: Sociology Press.

Glaser, B. (1998). Doing grounded theory: Issues and discussions. Mill Valley, CA, USA: Sociology Press.

Hartson, R. (2003). Cognitive, physical, sensory and functional affordances in interaction design. Behaviour \& Information Technology, 22(5), 315-339.

Hasan, H., Gould, P., Larkin, P., \& Vrazalic, L. (Eds.). (2001). Information systems and activity theory: Vol. 2, Theory and practice. Wollongong, Australia: University of Wollongong Press.

Hutchins, E. (1995). Cognition in the wild. Cambridge, MA, USA: MIT Press.

Hutchins, E. (2000). Distributed cognition. In N. J. Smelser \& P. B. Baltes (Eds.), The international encyclopedia of the social and behavioral sciences [online]. Retrieved June 10, 2000, from http://eclectic.ss.uci.edu/ drwhite/Anthro179a/DistributedCognition.pdf

Jones, P., Chu, R., \& Mitchell, C. (1995). A methodology for human-machine systems research: Knowledge, engineering, modeling and simulation. IEEE Transactions on Systems, Man, and Cybernetics, 25(7), 1025-1037.

Kaptelinin, V. (1996). Computer-mediated activity: Functional organs in social and developmental contexts. In B. A. Nardi (Ed.), Context and consciousness: Activity theory and human-computer interaction (pp. 45-68). Cambridge, MA, USA: Massachusetts Institute of Technology. 
Kontogiannais, T. (1996). Stress and operator decision making in coping with emergencies. International Journal of Human-Computer Studies, 45, 75-104.

Kuutti, K. (1996). Activity theory as a potential framework for human-computer interaction research. In B. A. Nardi (Ed.), Context and consciousness: Activity theory and human-computer interaction (pp. 17-44). Cambridge, MA, USA: Massachusetts Institute of Technology.

Lehane, P. (2008, November). Usefulness emergent in the user experience: An empirical and theoretical analysis of technology acceptance in the workplace. Paper presented at HC 2008, EACE and ACM International Conference, Nagaoka, Japan.

Lehane, P. (2010). Designing interactions for the collective user experience. In Proceedings of the 22nd Conference of the Computer-Human Interaction Special Interest Group of Australia on Computer-Human Interaction (OZCHI 2010; pp. 120-127). New York, NY, USA: ACM. doi: 10.1145/1952222.1952247

Lehane, P. (2012a). The system acceptance indicator: An analysis practice emergent from the plans, scripts and intuitive interaction of the collective user community (Unpublished doctoral dissertation). University of South Australia, Adelaide, Australia.

Lehane, P. (2012b). Make it intuitive: An evaluation of interaction design using plans and scripts from the computer community of practice. Human Technology: An Interdisciplinary Journal on Humans in ICT Environments, 8(1), 77-103.

Lehane, P., \& Huf, S. (2005). Towards understanding system acceptance: The development of an assessment instrument in practice. In Proceedings of the 17th Australia Conference on Computer-Human Interaction (OZCHI '05; pp. 1-9). Narrabundah, Australia: Computer-Human Interaction Special Interest Group of Australia.

Lehane, P., \& Huf, S. (2006). Designing interaction: Reduction in activity time by the re-allocation of user tasks to machine functions. In Proceedings of the 18th Australia Conference on Computer-Human Interaction (OZCHI '06; pp. 449-453). Sydney, Australia: Computer-Human Interaction Special Interest Group of Australia.

Lehane, P., \& Huf, S. (2007). Re-establishing user perception norms: How long does it really take? In Proceedings of ECCE 2007, EACE and ACM International Conference Proceedings Series (pp. 207-214). London, England: ACM.

Nardi, B. A. (Ed.). (1996a). Context and consciousness: Activity theory and human-computer interaction. Cambridge, MA, USA: Massachusetts Institute of Technology.

Nardi, B. A. (1996b). Studying context: A comparison of activity theory, situated action models, and distributed cognition. In B. A. Nardi (Ed.), Context and consciousness: Activity theory and human-computer interaction (pp. 69-102). Cambridge, MA, USA: Massachusetts Institute of Technology.

Nielsen, J. (1993). Usability engineering. San Diego, CA, USA: Morgan Kaufman.

Norman, D. (1988). The design of everyday things. New York, NY, USA: Doubleday.

Norman, D. (2004). Emotional design: Why we love (or hate) everyday things. New York, NY, USA: Basic Books.

Pawlak, W., \& Vicente, K. (1996). Inducing effective operator control through ecological interface design. International Journal of Human-Computer Studies, 44, 653-688.

Preece, J., Rogers, Y., \& Sharp, H. (2002). Interaction design: Beyond human-computer interaction. New York, NY, USA: Wiley.

Preece, J., Rogers, Y., \& Sharp, H. (2007). Interaction design: Beyond human-computer interaction (2nd ed.). New York, NY, USA: Wiley.

Preece, J., Rogers, Y., Sharp, H., Benyon, D., Holland, S., \& Carey, T. (1994). Human-computer interaction. Workingham, England: Addison Wesley.

Rasmussen, J. (1994). Cognitive systems engineering. New York, NY, USA: John Wiley \& Sons Inc.

Shiizuka, H. (2007). Overview of Kansei system and related problems. In J. T. Yao, P. Lingras, W.-Z. Wu, M. Szczuka, N. J. Cercone, \& D. Ślęzak (Eds.), Lecture Notes in Computer Science, Vol. 4481: Rough sets and knowledge technology (pp. 203-210). Berlin, Germany: Springer-Verlag. 
Shneiderman, B. (1982). The future of interactive systems and the emergence of direct manipulation. Behaviour and Information Technology, 1, 237-256.

Shneiderman, B. (1983). Direct manipulation: A step beyond programming languages. IEEE Computer, 16, 57-62.

Stammers, R., \& Shepherd, A. (1995). Task analysis. In J. Wilson \& N. Corlett (Eds.), Evaluation of human work: A practical ergonomics methodology (2nd ed.; pp. 144-168). Philadelphia, PA, USA: Taylor \& Francis.

Suchman, L. (1987). Plans and situated actions. Cambridge, MA, USA: Cambridge University Press.

Taylor, F. (1911). The principles of scientific management. New York, NY, USA: Harper Brothers.

Venkatesh, V., Morris, M., Davis, G., \& Davis, F. D. (2003). User acceptance of information technology: Toward a unified view. MIS Quarterly, 27(3), 425-478.

Vicente, K. (1999). Cognitive work analysis. Mahwah, NJ, USA: Lawrence Erlbaum Associates Inc.

Vygotsky, L. (1978). Mind in society: The development of higher psychological processes. Cambridge, MA, USA: Harvard University Press.

\section{Author's Note}

The SAI and an Excel worksheet with notes on use are available for use by those assessing the introduction of technology into the workforce (https://eportfolio.usq.edu.au/view/view.php?t=Zw1TAYBxKMOG5fUPunyi). The only prerequisite for the use of the SAI or the inherent analysis practice is that any published report should acknowledge the source of the measure and practice.

All correspondence should be addressed to

Pat Lehane

P.O. Box 32

Darling Heights

Queensland Australia 4350

pat.lehane@usq.edu.au

Human Technology: An Interdisciplinary Journal on Humans in ICT Environments ISSN 1795-6889

www.humantechnology.jyu.fi 